\title{
Prácticas Educativas Telepresenciales En Tiempos De La Pandemia Covid -19: Esquemas De Referencia De Docentes Y Estudiantes
}

\section{Telepresence Educational Practices In Times Of The Covid-19: Reference Schemes For Teachers And Students}

\section{(i) Luz Angela Cardona Arce ${ }^{1}$ (1) Julián David Vélez Carvajal ${ }^{2}$}

Recepción: Abril 9 de 2021 Aprobación: Mayo 18 de 2021 Publicación: Junio 30 de 2021

Cómo citar este artículo: Cardona A, Luz Angela. Vélez C, Julián (2021). "Prácticas Educativas Telepresenciales en tiempos de la pandemia COVID -19: Esquemas de referencia de docentes y estudiantes".

Miradas, Vol. 16, $\mathrm{N}^{\mathrm{o}}$ 1. pp. 9 - 30 https://doi.org/10.22517/25393812.24858

\section{Resumen}

Esta investigación tiene como propósito dar cuenta de los esquemas de referencia construidos por docentes y estudiantes de instituciones educativas públicas y privadas, de los departamentos de Risaralda, Quindío, Córdoba, Cundinamarca, Valle del Cauca, Bolivar, Santander, Huila y Antioquia en Colombia. Teóricamente se trabajó la categoría de esquemas observacionales desde

1 PhD Ciencias de la Educación, Docente Institución Educativa Suroriental, Pereira. ORCID: https://orcid.org/0000-0003-0631-7853. luancardona@colegiosuroriental.edu.co

$2 \mathrm{PhD}$ Ciencias de la Educación, Docente titular, Facultad de Comunicación Social - Periodismo, Universidad Pontificia Bolivariana, Montería, julian.velezc@upb.edu.co 
los desarrollos teóricos de Heinz Von Forester y Niklass Luhmann, igualmente, se abordaron las categorías prácticas educativas, usabilidad y competencias TIC desde la relación entre comunicación y cultura. Metodológicamente, la investigación tuvo un enfoque cualitativo. Como principal resultado se plantea lo siguiente: tanto estudiantes como docentes, construyen un esquema observacional de carácter relacional que se estructura a partir de las formas práctica educativa, competencias TIC, usabilidad TIC y comunicación / interacción / diálogo; esta última adquiere importancia por cuanto funge como enlace entre las demás.

En el caso específico de los docentes y estudiantes abordados en este proyecto de investigación, emerge un esquema observacional de carácter relacional que se estructura a partir de las siguientes formas: a) práctica educativa y TIC, b) competencias TIC, c) usabilidad TIC y d) comunicación / interacción / diálogo.

Palabras clave: Habilidades, mediación TIC, práctica educativa, usabilidad, competencias TIC, comunicación, interacción, telepresencia, esquemas observacionales.

\section{Abstract}

This research aims to account for the reference schemes built by teachers and students of public and private educational institutions in the departments of Risaralda, Quindío, Córdoba, Cundinamarca, Valle del Cauca, Bolivar, Santander, Huila, and Antioquia in Colombia. Theoretically, the category of observational schemes worked from the theoretical developments of Niklas Luhmann; Likewise, the categories of educational practices, usability and ICT skills were addressed from the relationship between communication and culture. Methodologically, the research had a qualitative approach. As the main result the following is proposed: both students and teachers build an observational scheme of a relational type that is structured from the forms of educational practice, ICT skills, ICT usability and communication / interaction / dialogue; the latter of them acquires importance because it acts as a link between the others.

For the specific case of teachers and students addressed in this research project, an observational scheme of a relational nature emerges and that is structured from the following ways: a) educational practice and ICT, b) ICT skills, c) ICT usability and d) communication / interaction / dialogue.

Keywords: skills, ICT mediation, educational practice, usability, ICT skills, communication, interaction, telepresence, observational schemes.

\section{Introducción}

Este artículo tiene como propósito dar cuenta de los esquemas de referencia con respecto a las prácticas educativas en medio de la pandemia COVID - 19, por parte de docentes y estudiantes de instituciones educativas públicas y privadas de las regiones de Risaralda, Quindío, Córdoba, Cundinamarca, Valle del Cauca, Bolivar, Santander, Huila y Antioquia en Colombia. La situación de salud pública que se ha tenido que enfrentar desde marzo de 2020, supuso un cambio abrupto en los procesos educativos, por cuanto el confinamiento decretado por los gobiernos nacional y local, implicó 
incorporar la telepresencia como la única alternativa para evitar una parálisis en la educación.

Bajo este panorama, las tecnologías digitales se sobrevaloraron a consecuencia de su utilidad, para enfrentar la situación causada por la pandemia, lo cual se sumó a la ausencia de un proceso de transición que mitigara los efectos del carácter abrupto de las nuevas medidas y que, por tanto, permitiera generar confianza en todos los actores involucrados en el proceso educativo. Este escenario provocó muchas confusiones, destacándose la sensación de lejanía entre la educación presencial y la mediada por tecnologías digitales.

Para enfrentar esta situación, las instituciones educativas iniciaron procesos de capacitación $\mathrm{y}$ de acompañamiento a los docentes, con el propósito de mitigar los traumatismos generados por las situaciones antes descritas; lo cual, en principio, generó las condiciones para que tanto docentes como estudiantes, pudieran avanzar en los procesos de enseñanza y aprendizaje. Pero ¿Cuáles son las expectativas y puntos de vista de los docentes $\mathrm{y}$ estudiantes frente a este nuevo escenario? ¿Bajo qué parámetros abordaron esta nueva situación?

Se considera importante abordar estos aspectos, por cuanto permiten acceder a información importante para tomar decisiones con respecto a las prácticas educativas no sólo para el presente, sino para proyectarlas al futuro, sobre todo si se tiene en cuenta que, probablemente, muchos de los aspectos incorporados en este coyuntura de telepresencia, se conviertan en estrategias permanentes del proceso educativo.

Por tanto, se hace necesario abordar los aspectos que, según los docentes y los estudiantes, constituyen las prácticas educativas en medio de la situación actual, caracterizada por las mediaciones tecnológicas para llevar a cabo la telepresencia. Lo cual permite generar reflexiones sobre la relación entre tecnologías digitales y educación, que permitan superar los enfoques instrumentales y vislumbrar oportunidades para la educación en las que se potencien las competencias de docentes y estudiantes, de tal manera que puedan generar procesos significativos en el marco de la relación tecnologías - educación.

En este orden de ideas, se planteó la siguiente pregunta de investigación ¿Cuáles son los esquemas de referencia construidos por los docentes y los estudiantes de instituciones educativas públicas y privadas de Risaralda, Córdoba y Antioquia, con respecto a las prácticas educativas en el marco de la telepresencia causada por la pandemia COVID - 19?

Como objetivo general se propuso: Distinguir los esquemas de referencia construidos por parte de los docentes y los estudiantes de instituciones educativas públicas y privadas de Risaralda, Córdoba y Antioquia con respecto a las prácticas educativas en el marco de la telepresencia causada por la pandemia COVID - 19. 
El artículo, expone estudios referentes a las reflexiones sobre la pandemia COVID-19 y la educación, aunque son pocos por lo reciente del tema; así mismo se presentan algunas categorías conceptuales que se usaron en la lectura de la información; luego, se presenta la metodología; finalmente, los resultados y su discusión.

Esperamos haber reportado datos para evaluar el proceso educativo con telepresencia, en tiempos de la pandemia COVID-19.

\section{Estado del arte y Marco teórico}

A consecuencia de la pandemia COVID - 19, estamos inmersos en un contexto en el que las mediaciones tecnológicas son de uso casi obligatorio en el ámbito educativo, productivo y social. En lo que respecta a la educación, los avances en ciencia y tecnología, han permitido que la sociedad contemporánea cuente con un acervo digital amplio que puede utilizarse en los procesos de formación para promover la reflexión, la formación, el intercambio y la discusión científica (Pardo Kuklinski, Hugo; Cobo, Cristóbal, 2020).

Lo anteriormente planteado permite inferir que a la par de la ampliación del potencial comunicativo para la telepresencia, también se hacen visibles brechas sociales $\mathrm{y}$ educativas que se materializan en un acceso desigual a las tecnologías y la conectividad; lo cual se traduce en una distribución inequitativa de los recursos y de las estrategias, afectando, principalmente, a sectores de menores ingresos y mayor vulnerabilidad (Rieble-Aubourg y Viteri, 2020).

En lo que respecta a la educación en los países en vía de desarrollo, la pandemia COVID - 19, ha implicado toda una serie de reflexiones en torno a las acciones educativas, sobre todo con relación a los contextos de implementación del currículo, no solo por la articulación de herramientas tecnológicas en un contexto que plantea condiciones diferentes para la ejecución del currículo, sino, también, porque algunos aprendizajes y competencias cobran mayor relevancia en el actual contexto (CEPAL, N., 2020).

En este orden de ideas, se reflexiona acerca de cómo la "necesidad", asociada a las disrupciones que impone la pandemia, ha involucrado a todos los actores del proceso educativo en prácticas para continuar y avanzar en la formación dadas las características de sus poblaciones, mismas que, en muchas ocaciones, no cuentan con las condiciones socioeconómicas favorables para garantizar su participación en la educación por telepresencia, puesta a disposción por las instituciones educativas en todos los niveles de formación; lo cual ha implicado que los centros educativos hayan movido su oferta educativa hacia una formación a distancia facilitada por la tecnología educativa, en la que los profesores gestionan diversas estrategias para llegar a los estudiantes y mantenerlos activos en el proceso, lo cual puede afectar la inclusión de los estudiantes. (CEPAL, N., 2020). 
En este contexto, se producen importantes reflexiones acerca de los cambios de los que fueron objeto las dinámicas de formación, los cuales podrían servir como punto de partida para grandes transformaciones que se han proyectado desde tiempos pasados y que hoy empiezan a materializarse, pues se posicionan como la única alternativa para no clausurar las actividades educativas; lo cual ha implicado un mayor nivel de tolerancia hacia el error, por parte de los estudiantes, en las prácticas educativas (Pardo Kuklinski, Hugo; Cobo, Cristóbal, 2020).

En este sentido, se puede evidenciar que las prácticas educativas en el marco de la pandemia, se improvisan y, por tanto, movilizan gran parte de las experiencias del aula física, al ciberespacio (Hodges et al., 2020), lo cual ha implicado la creación de nuevos diseños, de tal manera que las experiencias de formación se realicen de forma exclusiva en plataformas digitales, como un movimiento impuesto por la COVID-19.

En cuanto a los diversos escenarios de formación afectados por la situación de confinamiento, se establece que las prácticas educativas, en general, han vinculado procesos de mediación tecnológica como única posibilidad para avanzar en la formación de los estudiantes, lo cual puede derivar en el favorecimiento de sus habilidades de pensamiento, con respecto a la gestión de las actividades cotidianas en los diversos entornos de aprendizaje a los cuales pertenecen. Es así como las habilidades de pensamiento de orden superior, se posicionan como un reto para que los estudiantes interpreten, analicen o manipulen información, dadas las facilidades que se pueden encontrar en las TIC a nivel de interacción y acceso remoto, lo cual incide en el favorecimiento de prácticas y experiencias, influyendo no solo en el alcance de los objetivos con respecto a los contenidos y al aprendizaje, sino, también, en el diseño y desarrollo de enfoques en los estudios y la práctica profesional, (Akhyar, M.,2020).

No obstante, la realidad tecnológica que afrontan los profesores en el contexto anteriormente descrito, trae consigo retos importantes, en los que las TIC pueden contribuir al desarrollo de habilidades tanto para ellos como para sus estudiantes. En este sentido, las concepciones de los profesores son uno de los factores que pueden limitar el uso que hagan de estas herramientas; en este sentido, se destaca que los profesores reconocen potencialidades de las TIC al considerar que tienen un efecto positivo en el aprendizaje $\mathrm{y}$ desarrollo de las habilidades del siglo XXI en sus estudiantes; dichos beneficios se observan en factores como la comunicación, la colaboración y el pensamiento crítico, entre otros. Por lo tanto, el rol docente en el entorno comunicacional digital, demanda la necesidad promover la integración de las TIC en un modelo pedagógico en el que la formación de profesores en habilidades digitales se demuestra en el contexto del pensamiento de orden superior. (Liesa-Orús, M., LatorreCosculluela, C., Vázquez-Toledo, S., \& Sierra-Sánchez, V.,2020). 
Conviene resaltar los aportes de la aplicación de estrategias en las que se vincula la mediación tecnológica, pues logran favorecer el desarrollo de habilidades como las mencionadas hasta el momento, a partir de la implementación de estrategias basadas en problemas, en el aprendizaje colaborativo; entre otras (Oseda Gago, Dulio, Mendivel Geronimo, Ruth Katherine, \& Angoma Astucuri, Miriam., 2020).

De forma similar, el uso de herramientas tecnológicas imprime, actualmente, una forma específica para determinar el producto que se obtiene a partir de la transformación de la información en conocimiento científico $\mathrm{y}$, de este último, en innovación investigativa, para los escenarios de formación, en el contexto actual de la COVID-19, (Cruz Pérez \& Pozo Vinueza, 2020), lo cual implica que dichos procesos conllevan al uso de las TIC para la obtención de datos e información, la cual no se limita a la utilización de las tecnologías, sino a un enfoque de estrategias educativas orientadas a las acciones autónomas para la búsqueda, intercambio, reflexión y análisis de la información que permita, a los sujetos, la generación de conocimiento útil en contextos específicos. (Cruz Pérez \& Pozo Vinueza, 2020)

Comoresultado de la adecuación de los estándares en mención, se sugieren 18 competencias para los profesores, mismas que se organizan en torno a seis aspectos de su práctica profesional: a) comprensión del papel de las TIC en las políticas educativas; b) currículo y evaluación; c) pedagogía;

d) aplicación de competencias digitales;

e) organización y administración; y f) aprendizaje profesional de los docentes. En síntesis, dichas competencias, se agrupan en tres niveles del uso pedagógico de las TIC, por parte de los docentes: adquisición, profundización y creación de nuevos conocimientos (UNESCO, 2019).

En este sentido, el escenario de la pandemia COVID-19, trajo consigo una amplia promoción e implementación de cursos en línea, webinars gratuitos para docentes, que se han concentrado en el desarrollo y mejora de habilidades digitales en el contexto de la educación virtual a distancia. Para el caso de Colombia, mediante el Plan Padrino, se ha buscado promover el fortalecimiento de capacidades y el intercambio de experiencias pedagógicas entre instituciones educativas en torno al uso y la apropiación de las TIC en los procesos formativos (Ministerio de Educación de Colombia, 2020).

En síntesis, las investigaciones anteriormente referidas, abordan diversas temáticas relacionadas con la articulación de las TIC a la educación, en el contexto de la pandemia COVID 19; dentro de las cuales, se destacan los siguientes aspectos:

- Avances tecnológicos y
científicos que permiten consolidar
la educación mediada por TIC.
- Brechas socio - económicas
en cuanto al acceso a las TIC, la
conectividad y la adquisición de
competencias digitales.


- Articulación de la TIC al currículo.

- Adaptación de la educación a las condiciones planteadas por la pandemia COVID - 19, sobre todo en lo que tiene que ver con la educación a distancia mediada por TIC.

- Fortalecimiento de las habilidades de pensamiento en estudiantes y docentes.

- Percepciones de los docentes con respecto al uso de las TIC.

Sin duda, dichos aspectos se hacen centrales al momento de reflexionar sobre la educación en el contexto de las condiciones de telepresencia que ha impuesto la pandemia, en el sentido de que su irrupción implicó que todas las instituciones educativas hicieran un tránsito abrupto hacia la educación mediada por TIC, proceso en el que no sólo se tuvo que hacer uso de las herramientas tecnológicas disponibles, también, se requirió capacitar a docentes y estudiantes para poder enfrentar esta situación.

Esta investigación contribuye a profundizar en los aspectos antes mencionados en la medida en que trata de comprender cuáles son los esquemas observacionales de los docentes con respecto a las prácticas educativas que se tuvieron que implementar a partir del confinamiento propiciado por la pandemia COVID - 19.
En cuanto a los conceptos que guiaron la lectura de los datos (marco teórico) referenciamos los más centrales.

En el 2020, a raíz de la pandemia COVID-19, el sistema educativo se enfrentó a un cambio abrupto, en los procesos de enseñanza-aprendizaje, por cuanto hubo que incorporar la telepresencia, como única alternativa para evitar suspender la educación formal.

Bajo ese contexto, nos dimos a la tarea de indagar, a profesores y estudiantes, sobre la percepción de la experiencia vivida, al incluir esa forma de abordar lo educativo. Pero más allá de esto, ahondamos en la información para encontrar los esquemas de observación profundo, a partir de lo que dijeron, que nos llevan a dar cuenta de lo emergente en esa experiencia, con el objetivo, de llamar la atención al respecto, e indicar aquellas prácticas que se pudieran llevar a la presencialidad, por su eficacia en el proceso de enseñanza-aprendizaje.

Así, entonces, nos valimos de los desarrollos teóricos de Heinz Von Forester (1970) y Niklas Luhmann (1995), sobre lacategoría de observación de segundo orden. Para los autores en mención, la observación consiste en un procesamiento altamente selectivo que opera a partir de la distinción entre actualidad y potencialidad, lo cual implica que, en dicho proceso, frente a la información disponible, los observadores seleccionan una porción de esta, dejando el resto sin procesar. La pregunta, es entonces, ¿Qué criterios se tienen en cuenta para realizar tal 
selección? La respuesta no está en el plano de lo consciente, sino en lo que dicen a través de lo que no dicen.

Las categorías de medio y forma, permiten comprender el proceso anteriormente descrito. El medio se entiende como una serie de unidades dispersas que son susceptibles de ser relacionadas de diferentes maneras por los observadores. Por otro lado, la forma se entiende como el resultado de las relaciones que asumen las unidades dispersas a partir de los procesos selectivos ejecutados por el observador (Luhmann, 1998). Al entender que todos somos observadores altamente selectivos, nos preguntamos sobre cuáles son las distinciones que hacen los docentes y estudiantes, abordados en esta investigación, con respecto a las prácticas educativas.

Así mismo, conceptos como práctica educativa (Zabala, 1995), que resalta la complejidad humana y técnica en la educación mediada por TIC; la usabilidad Cobo(2009) que apunta a comprender las implicaciones socioculturales de las tecnologías digitales más allá del enfoque instrumental; Castells (2014) introduciendo en la usabilidad, las interacciones y usos sociales; como Lévy (2004), que coloca en énfasis de este concepto, en el contexto compartido para aprender, lo que implica transformar continuamente el universo virtual dentro del cual toman sentido; las competencias en TIC, propuesta por la UNESCO (2019) que priorizan la formación docente en tecnología comunicación, pedagogía, investigación y gestión trasversalizadas por dichas herramientas, nos permitieron comprenden el ámbito en que se enmarca el estudio que precede a éste artículo, por lo premonitorio de los mismos, en la época del COVID 19.

En términos generales, las relaciones dinámicas que surgen en los entornos comunicacionales, todas ellas fungen en difusión de manera continua desde el entorno de medios, al desarrollo de habilidades, así como también del centro al entorno comunicacional, para transformar el paisaje móvil de significaciones de los colectivos que participan en las prácticas educativas, donde las habilidades se producen en la interacción entre los pensamientos de los sujetos y el contexto sociocultural, (Csikszentmihalyi, M.,1998).

\section{Metodología}

Esta investigación es de carácter cualitativo; en tal sentido, se asume una posición filosófica que considera a la realidad como una construcción social, en la que el lenguaje nos permite exteriorizar lo que pensamos; por lo que este nos sirve como medio para comprender dicha realidad. Por lo tanto, cuando abordamos los datos desde un punto de vista cualitativo, tenemos como propósito dar cuenta de las motivaciones y fundamentos socio - culturales desde los cuales los sujetos actúan e interpretan el mundo. (Bedoya, 2019). En este caso, abordamos los esquemas de referencia de los entrevistados. 


\section{Población y muestra}

La población se encuentra constituida por docentes de universidades, instituciones educativas de educación básica primaria y secundaria, así como también de la formación técnica, a nivel nacional de las ciudades de Pereira, Monteria, Medellín, Buga, Cali, Cartagena, Florida Blanca, Tulua, Santa Rosa, Bogotá, Armenia, Neiva, Villanueva Bolivar, Cartago y a nivel internacional de Canadá, Spriengfield, Guatemala, Perú, México, Ecuador, Venezuela.

Todas las personas seleccionadas, cumplieron con el criterio de selección relacionado con el ejercicio de prácticas educativas por telepresencia en el marco del confinamiento causado por la pandemia COVID - 19. Igualmente, fueron beneficiarios de capacitaciones en competencias TIC y tuvieron acceso a diversas plataformas digitales para el desarrollo de sus clases. Se seleccionó una muestra no probabilística de 136 docentes y 176 estudiantes en total.

\section{Recolección de la información}

Para la recolección de la información, se implementó un formulario autoadministrable en línea a través de las herramientas de Google. Para su diseño, se tuvieron en cuenta las siguientes categorías:
Tabla 1. Categorías Formulario Autoadministrable

\begin{tabular}{|c|c|}
\hline Categorías & Subcategorías \\
\hline \multirow[t]{3}{*}{ Experiencia Docente } & $\begin{array}{l}\text { Formación en TIC para } \\
\text { el desarrollo de procesos } \\
\text { educativos }\end{array}$ \\
\hline & $\begin{array}{c}\text { Uso de herramientas } \\
\text { tecnológicas en actividades } \\
\text { laborales }\end{array}$ \\
\hline & $\begin{array}{c}\text { Aporte de las herramientas } \\
\text { tecnológicas a las actividades } \\
\text { cotidianas }\end{array}$ \\
\hline \multirow{4}{*}{$\begin{array}{l}\text { Reconocimiento de } \\
\text { Prácticas Educativas }\end{array}$} & $\begin{array}{c}\text { Apropiación de las TIC en las } \\
\text { prácticas educativas }\end{array}$ \\
\hline & $\begin{array}{c}\text { Estrategias enseñanza/ } \\
\text { aprendizaje implementadas en } \\
\text { la telepresencia }\end{array}$ \\
\hline & $\begin{array}{c}\text { Metodologías de enseñanza - } \\
\text { aprendizaje implementadas en } \\
\text { la telepresencia. }\end{array}$ \\
\hline & $\begin{array}{c}\text { Dificultades en la práctica } \\
\text { educativa }\end{array}$ \\
\hline \multirow{4}{*}{$\begin{array}{l}\text { Elementos de la } \\
\text { práctica educativa }\end{array}$} & $\begin{array}{l}\text { Recursos tecnológicos en } \\
\text { procesos de acompañamiento a } \\
\text { estudiantes }\end{array}$ \\
\hline & $\begin{array}{c}\text { Fortalecimiento de las } \\
\text { actividades educativas a partir } \\
\text { de estrategias mediadas por } \\
\text { TIC }\end{array}$ \\
\hline & $\begin{array}{l}\text { Interacciones que se potencian } \\
\text { en la práctica educativa. }\end{array}$ \\
\hline & $\begin{array}{c}\text { Habilidades que se } \\
\text { fortalecen con las estrategias } \\
\text { implementadas en la } \\
\text { telepresencia }\end{array}$ \\
\hline
\end{tabular}

\section{Análisis de la información}

Para el análisis de la información se realizó bajo los parámetros del enfoque cualitativo, por tanto, se implementó el siguiente procedimiento, que en un primer momento utilizó el software Orange como filtro de representatividad de la información de las respuestas: 


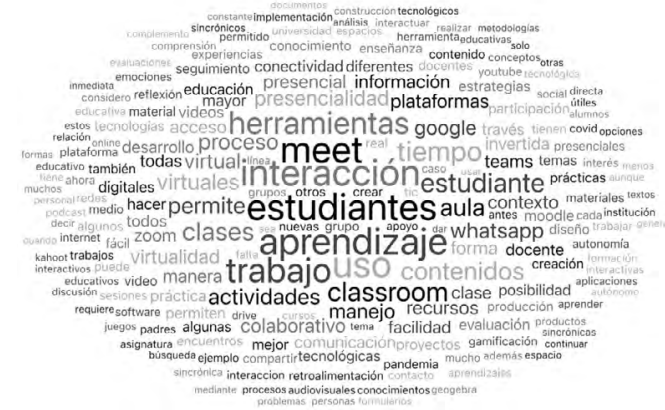

Nota: Fuente propia

Luego se continuó con:

a) Agrupación de respuestas por categorías predefinidas.

b) Establecimiento de relaciones entre los diferentes grupos establecidos.

c) Nominación de agrupaciones para el establecimiento de categorías emergentes.

d) Elaboración de esquema categorial.

\section{Consideraciones éticas}

Según las normatividades de las instituciones implicadas, dadas sus características, este proyecto de investigación no requiere aval por parte del comité de ética de las instituciones involucradas en este proyecto. Sin embargo, la investigación se desarrolló fundamentándose en los derechos de las personas involucradas según lo establecen las leyes colombianas.

\section{Descripción de resultados}

Se presentan los datos obtenidos como resultado en la implementación de instrumentos, con la población de docentes y estudiantes que participaron del estudio:

\section{Profesores}

Figura 1. Experiencia de Formación TIC

Profesores

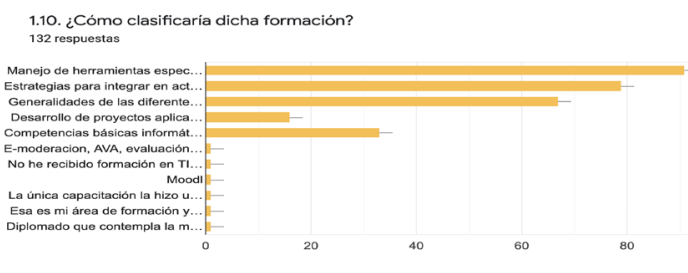

En las experiencias de formación TIC del docente, se hace referencia a los aspectos técnicos asociados al manejo de herramientas; sin embargo, se observa una valoración de las estrategias que podrían integrarse a las actividades de aprendizaje del contexto educativo. En ese sentido, se evidencia una relación entre el manejo básico de las herramientas (Manejo de herramientas específicas, generalidades de las diferentes herramientas disponibles, competencias básicas informáticas) y la apropiación de las mismas para los procesos pedagógicos de enseñanza - aprendizaje (Desarrollo de proyectos aplicados al contexto, estrategias para integrar en actividades de aprendizaje). 
Figura 2. Condiciones para Vincular herramientas tecnológicas en actividades laborales del docente

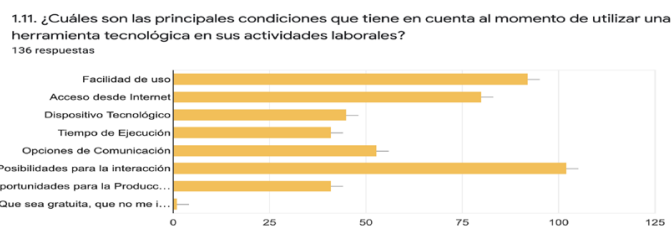

Los docentes presentan algunas condiciones que tienen en cuenta para la vinculación de algún medio tecnológico en las actividades laborales, hacen énfasis en aspectos como facilidad de uso, acceso desde internet y posibilidades para la interacción. Con respecto a este último aspecto, se evidencia que "la posibilidad de interacción", resalta las oportunidades para el establecimiento del vínculo social, mismas que se relacionan con los procesos de intercambio para aprender con el otro; esto es relevante, si se tiene en cuenta que la interacción es algo que va más allá del acceso a las tecnologías, la conectividad y el manejo instrumental de las mismas.

Lo anteriormente planteado, se tecnológicos con relación a las situaciones de usos y alcances de objetivos específicos en las prácticas cotidianas.

También se da relevancia a la disponibilidad de "Acceso a y desde internet", lo cual se relaciona con aspectos como conectividad $y$ accesibilidad; a este aspecto se pueden relacionar las selecciones hechas con relación al dispositivo tecnológico y al tiempo de ejecución. De igual manera, el concepto de ubicuidad se posiciona como elemento fundamental en la transformación de los espacios y las formas de acceso, pues sólo se requiere disponer de cuentas asociadas a correo electrónico, en diversos dispositivos y lugares. En síntesis, se puede destacar que, en cuanto a las selecciones que hacen los entrevistados, hay paridad entre el acceso, el manejo instrumental de las tecnologías y los procesos de comunicación e interacción humana como aspecto central.

Figura 3. Aporte de las TIC en actividades de los profesores relaciona con el énfasis que hacen los docentes con respecto a las “opciones de comunicación”. Los procesos comunicativos cobran importancia, por cuanto permiten las cuales trabajan y, en general, para el intercambio de procesos académicos. Se observa, también, que la categoría "facilidad de uso", se relaciona con la adquisición de competencias digitales, así mismo, se puede inferir que la categoría usabilidad, se hace importante al momento de evaluar y tomar decisiones frente a los aspectos

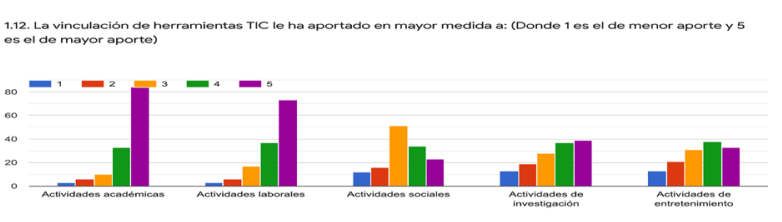

La vinculación tecnológica en actividades de los docentes, presenta relevancia en cuanto a las actividades académicas y laborales; a estas se les puede asociar, también, las actividades de investigación, dado que la población abordada pertenece al contexto educativo; que el valor otorgado a estos aprendizajes va más allá lo instrumental. 
Las actividades de entretenimiento y las actividades sociales, se ubican con valores inferiores, limitado tal vez al conservar la visión del encuentro social como esparcimiento libre, sin embargo, se podría pensar en lo social como elemento dinamizador de todas las otras actividades desarrolladas por los entrevistados. Esto se relaciona con el énfasis que hacen, en las otras preguntas, con respecto al componente comunicativo.

Figura 4. Estrategias de aprendizaje útiles en procesos de formación en la pandemia COVID-19

2.2. ¿Cuáles estrategias de aprendizaje considera que han sido más útiles en los procesos co formación en época de pandemia?

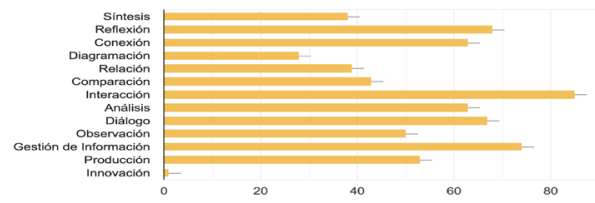

Se destaca que las estrategias de aprendizaje que alcanzan mayor reconocimiento son aquellas que se pueden llevar a cabo tanto en lo presencial como a través de las tecnologías digitales (reflexión, conexión, interacción, análisis, diálogo y gestión de la información). En este aspecto, también se observa que se eligieron dos estrategias que, de manera explícita, hacen referencia a la relación con los demás: interacción y diálogo. También es importante la referencia a las estrategias de relación, comparación, observación, producción, diagramación; es posible que haya una relación de interdependencia entre estas estrategias y las anteriores, por ejemplo, para hacer comparación es necesario el desarrollo de procesos de análisis entre otros.

Así, la interacción confirma la importancia asignada al intercambio con las demás personas. También los procesos asociados a la reflexión se ubican como una estrategia importante en el proceso de formación en tiempos de pandemia, dadas las posibilidades para avanzar en construcciones colectivas e individuales desde las diferentes concepciones de mundo en contextos socioculturales dinámicos.

Por otro lado, se evidencia el diálogo como una estrategia que permite el reconocimiento de los otros en un proceso de aprendizaje permanente, la conexión pone en escena la oportunidad de movilizar las estrategias al concepto de red de aprendizaje, para fortalecer algunas que habilitarán a los sujetos para navegar, extender y configurar redes personales de aprendizaje que trascienden en colectivos con intereses comunes.

Es importante conocer estas estrategias en la medida en que permiten tener una imagen desde el rol del docente y la manera en que vincula estrategias para aprender en sus experiencias personales de formación, ésto como punto de partida al momento de generar y diseñar estrategias para los estudiantes, teniendo en cuenta algunos elementos de su ruta personal en el vínculo social. Finalmente, la habilidad de la gestión de la información ubica el entorno comunicacional en el cual se movilizan las prácticas de formación, donde es indispensable establecer relaciones constructivas con la 
información que circula en volúmenes y velocidades exponenciales, la autorregulación, discriminación de la misma, comprensión de lógicas algorítmicas básicas que permiten una disposición cognitiva superior al estar expuestos en dichos entornos comunicacionales.

En todo caso, al tener en cuenta el contexto de pandemia, se evidencia que los docentes entrevistados ven en el uso de las tecnologías digitales un potencial importante para desarrollar estrategias y capacidades de pensamiento en los procesos educativos. Esto es interesante si se relaciona con las condiciones de uso de TIC y aporte de las TIC en las actividades laborales de los docentes, pues en ellas se hace referencia prevalente a los procesos de interacción y a las actividades académicas, investigativas y laborales, lo cual es coherente con lo que se señala en esta pregunta. En síntesis todas las estrategias se ubican como índices de la insistencia en la comunicación con el otro y muchos de los otros aspectos, se pueden leer como actividades que las personas pueden realizar en forma individual.

Figura 5. Metodologías implementadas en las prácticas educativas durante la COVID 19

Metodologías implementadas en prácticas educativas durante la COVID 19

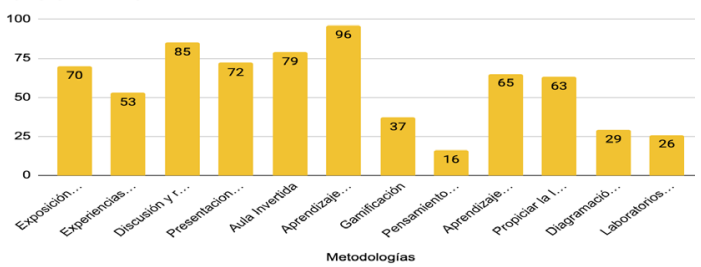

Es importante hacer referencia a las metodologías implementadas en las experiencias de aplicación en contexto, se evidencia que, en general, se da relevancia a las relaciones con los demás, sin restar validez a los procesos individuales. Se destaca, también, la relevancia que se da a la magistralidad, lo cual indica que, a través de las tecnologías digitales, también se pueden realizar procesos que se llevaban a cabo en la presencialidad.

Igualmente, aspectos como presentaciones de los estudiantes, aula invertida y aprendizaje basado en problemas, son metodologías que también fueron ampliamente seleccionadas por los profesores y se caracterizan por el papel activo que le dan al estudiante y su interacción con los demás, lo cual refuerza el rasgo común establecido con la interacción, la comunicación y el diálogo. Por consiguiente, se evidencia que el aprendizaje colaborativo expone la importancia de la experiencia social entre los sujetos que aprenden; lo mismo sucede con la discusión y la reflexión, pues con ambas se propicia la interacción.

Estos resultados apuntan al reconocimiento de dinámicas cíclicas que propicien el vínculo social a través de la colaboración, la solución de problemas en colectivo y la aplicabilidad de aprendizajes en experiencias cercanas, todo lo cual confirma la importancia de los otros con respecto al aprendizaje. A partir de la interacción, la comunicación y el diálogo, se fortalecen las relaciones presentes en las prácticas educativas. 
Figura 6. Habilidades que se fortalecen con las estrategias implementadas durante la COVID-19

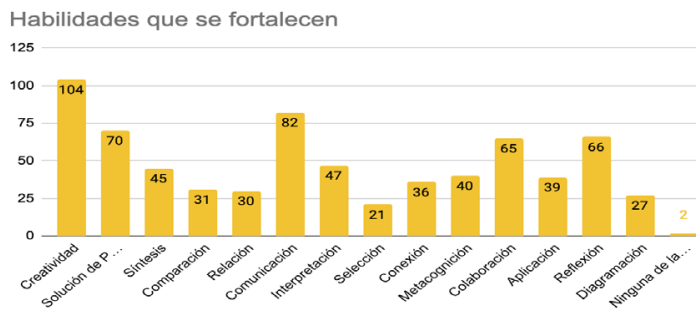

Las habilidades más destacadas por los profesores son la creatividad, solución de problemas, comunicación, colaboración y reflexión, donde se presenta la comunicación como uno de los aspectos más relevantes que favorece la vinculación de las estrategias colaborativas y reflexivas para el fortalecimiento de habilidades creativas en los ambientes mediados por tecnología e información. Es recurrente la relación marcada en cuanto a aspectos tales como interacción, comunicación, colaboración y conexión con el contexto en los datos analizados en el instrumento.

Figura 7. Interacciones que se potencian en los estudiantes con las nuevas prácticas educativas

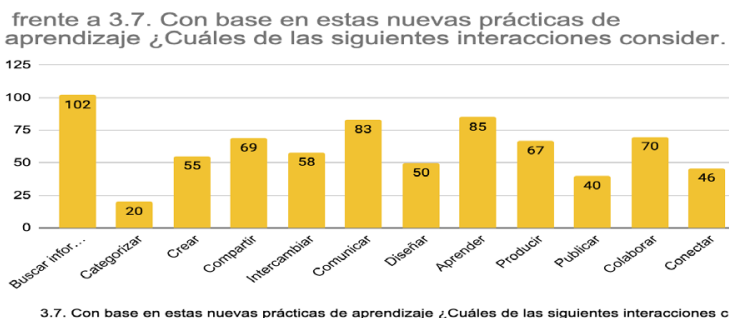

En cuanto a las interacciones que se potencian en los estudiantes, se da relevancia a las siguientes: buscar información, comunicar y aprender a partir de su recurrencia recíproca en las redes de aprendizaje que se constituyen en los procesos de formación mediada antes y durante la pandemia. Nuevamente la comunicación se hace protagonista, como una de las interacciones más visibles, dinámicas y aglutinantes, para los encuentros e intercambios entre los sujetos, a partir de las diferentes tecnologías. Se mantiene el rasgo común de los datos analizados hasta este momento, donde comunicar e interactuar aparecen con valoración importante por parte de los profesores en las prácticas de aprendizaje mediadas por TIC y se mantiene la tendencia en las interacciones de evocar la relación con el otro.

\section{Estudiantes}

Figura 8. Condiciones que tienen los estudiantes para seleccionar herramientas para las clases

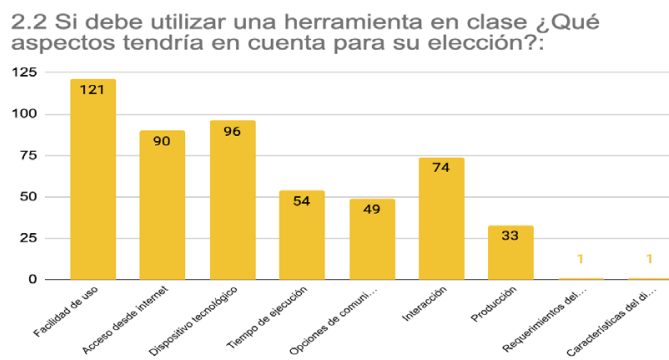

Desde la perspectiva de los estudiantes las condiciones más importantes a la hora de utilizar una herramienta tecnológica en sus clases, son el acceso desde internet, fácil uso y el dispositivo tecnológico. Es interesante el reconocimiento a la condición que permite movilidad en 
términos de ubicuidad, donde las herramientas ofrecen posibilidades para acceder a ellas desde internet a partir de cuentas de correo electrónico, es decir, la concepción de contar con dichas aplicaciones y sus datos en línea, sin limitaciones para su acceso en cualquier momento y en cualquier lugar.

Por otra parte, la condición que refiere la facilidad de uso, contiene algunas habilidades digitales individuales que orientan el juicio sobre la facilidad o no, de una herramienta tecnológica. Esa facilidad está contenida, también, en términos de la usabilidad, como uso consciente y con sentidos para el sujeto en la práctica específica para la cual la vincula. De esta manera, el dispositivo tecnológico se presenta como condición, que acompañen dicho acceso desde internet y la facilidad de uso, con las diversas posibilidades a nivel de dispositivos móviles, portátiles para el desarrollo de actividades asociadas a las clases en este contexto; no obstante, la interacción alcanza un nivel significativo en la percepción de los estudiantes, la cual podría funcionar en dichos escenarios como una condición que vincula a las otras condiciones en las acciones concretas de uso.
Figura 9. Habilidades que los estudiantes consideran se fortalecen en las clases mediadas por TIC durante la COVID-19

3.5 ¿Considera que las clases que ha recibido con la incorporación de las TIC, fortalecen algunas de las siguiente.

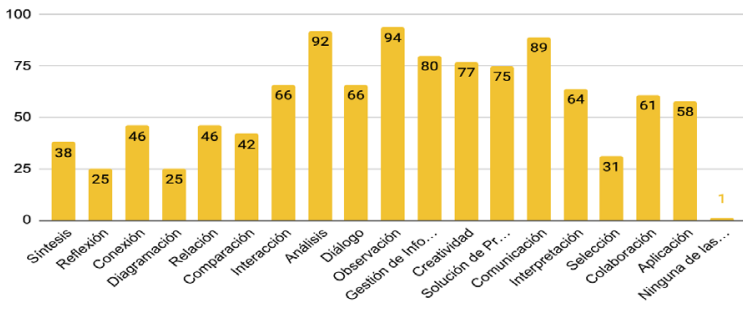

Los estudiantes señalan algunas de las habilidades que consideran fortalecidas en las clases mediadas por TIC, se observa el reconocimiento a la habilidad análisis, como un proceso del pensamiento que establece relaciones cognitivas importantes en las experiencias de aprendizaje, que permite el desarrollo de procesos inferenciales conscientes del sujeto en diversas experiencias, la observación como habilidad importante para la lectura del contexto, de las situaciones, datos, personas, a las cuales son expuestos los estudiantes en sus actividades para aprender.

También se refiere la comunicación como un enlace circulante entre las otras habilidades, para favorecer el vínculo social en las diversas modalidades de formación, estas habilidades son comunes en la presencialidad, donde se propende por mantener activa la interacción, para promover con ella el desarrollo de habilidades intelectuales.

La gestión de la información se presenta como una habilidad necesaria para la utilización de tecnologías 
digitales, las cuales abren posibilidades de manera continua a partir del acceso a la información, dicho acceso no es suficiente en los tiempos actuales, pues demanda habilidades como la "lectura entre líneas", validar fuentes, discriminar, clasificar, organizar, transformar, por tanto, la habilidad de gestión conlleva otras habilidades que se activan en las experiencias que así lo exijan.

Ahora bien, la creatividad se podría pensar como una habilidad emergente de las demás, donde el contexto sociocultural, la manipulación simbólica y el vínculo social, establecen conexiones con los individuos para ser propositivos en la transformación de prácticas, procesos, teorías, soluciones alternativas a problemas, etc.

Igualmente la reflexión, se considera una habilidad necesaria y activa en escenarios presenciales de cualquier tipo, así cómo también en los que vinculan las mediaciones tecnológicas. Dicha habilidad propicia en los individuos procesos del pensamiento que evocan posturas, concepciones de mundo, opiniones, argumentación, cuestionamientos, necesarios en los procesos educativos en cualquier nivel y área. De este modo, la mediación de herramientas tecnológicas, no es protagonista en estos escenarios, lo que se observa es la valoración a los usos en conexión con aspectos metacognitivos que los estudiantes desarrollan en las experiencias de formación en la relación con los otros. Todas las habilidades están interconectadas, se activan con las interacciones que se dan en el vínculo social de los ambientes en los cuales se movilizan los sujetos, donde la interacción movilizadora y circulante es la comunicación.

\section{Figura 10. Interacciones que los estudiantes} consideran útiles en el aprendizaje 4.3 ¿Cuáles de las siguientes interacciones considera usted
útiles en su aprendizaje?

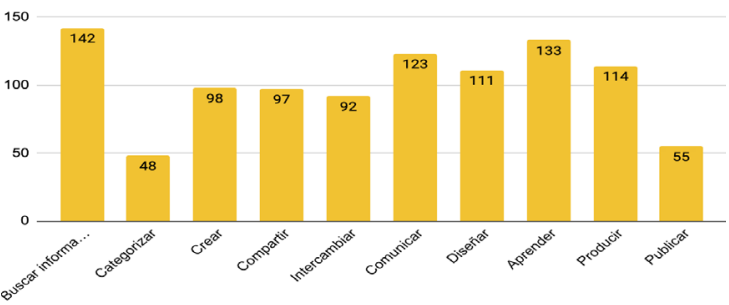

Muchas de las interacciones marcadas por los estudiantes, están asociadas a una o varias habilidades del pensamiento, allí está la acción de reciprocidad de las mismas, entre los nodos (sujetos, teorías, tecnología, información, estrategias...) que constituyen las prácticas educativas o escenarios de aprendizaje (presencial o mediado por TIC).

La interacción con mayor recurrencia entre los estudiantes es buscar información, la cual demanda de las habilidades de gestión de la información para acceder a fuentes diversas de la misma, habilitar criterios para su selección, validación, transformación y usos, se insiste en la evidencia en la relación fuerte entre interacción para el desarrollo de las habilidades en los enlaces que propicia la comunicación como interacción central.

Por otra parte, la interacción para aprender está cargada de significados y sentidos que encuentran los sujetos cada 
vez que realizan experiencias en los múltiples espacios que constituyen su ámbito sociocultural, en el cual aprende de manera permanente con otros en los enlaces que propicia la interacción comunicar, finalmente crear, producir y diseñar propician en los estudiantes la generación de una memoria común de datos, categorías, transformación de conocimiento tácito en explícito, en la valoración de los otros que acompañan las experiencias de interacción continua en los entornos comunicacionales.

\section{Discusión de resultados y conclusiones}

Figura 11. Esquema observacional de docentes y estudiantes sobre prácticas educativas

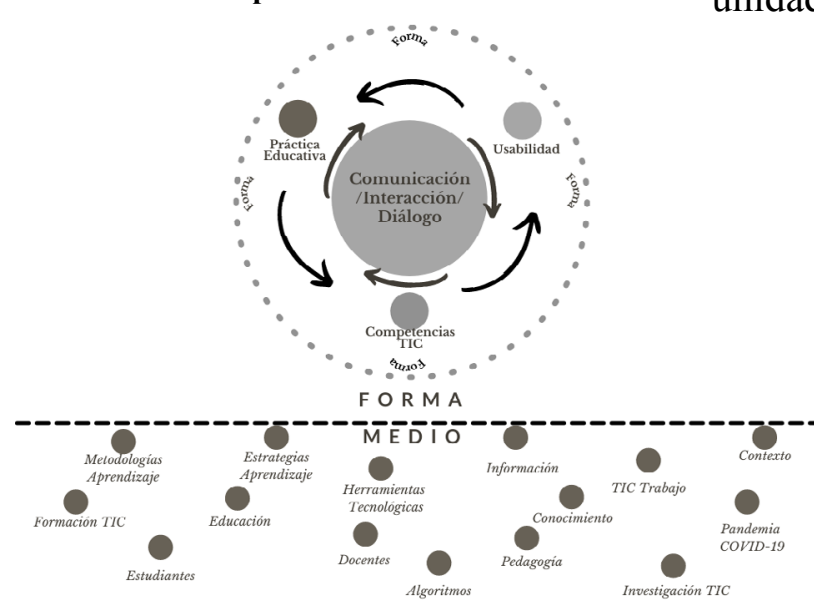

Según lo estipulado en la perspectiva teórica de este proyecto de investigación, se entiende que la observación consiste en un procesamiento de selecciones que opera a partir del par de oposición relevancia /opacidad (Luhmann, 1995). En este sentido, se hace pertinente la siguiente pregunta ¿Cuáles son los procesamientos selectivos que realizan los docentes y estudiantes entrevistados con respecto a las prácticas educativas en el marco de la pandemia COVID 19 ?

Para dar respuesta a esta interrogante, es importante hacer referencia a las categorías de medio y forma propuestas por el autor en mención (Luhmann, 1995), pues estas categorías nos permiten hacer operativa la manera en que los observadores realizan procedimientos selectivos. Al ser el medio una compilación de unidades dispersas que no oponen resistencia a las múltiples opciones de combinación que puede llevar a cabo un observador, nos preguntamos, entonces, acerca de cuáles son esas unidades que constituyen el medio para los docentes y estudiantes que fueron abordados en este proyecto de investigación.

En tal sentido, al indagarse sobre las prácticas educativas en el marco de la pandemia COVID-19, se puede establecer que las siguientes categorías, se constituyen en el medio a partir del cual los docentes y estudiantes abordados realizaron sus observaciones: 1) formación TIC, 2) estudiantes, 3) investigación TIC, 4) estrategias de enseñanza y aprendizaje, 5) docentes; 6) TIC y trabajo, 7) pedagogía, 8) herramientas tecnológicas, 9) metodologías de enseñanza / aprendizaje, 10) educación, 11) pandemia COVID - 19. 12) información, 13) conocimiento, 14) algoritmos, 15) contexto. 
Si las categorías anteriormente mencionadas fungen como unidades dispersas que se adaptan a las diferentes combinaciones que realizan los observadores, se hace pertinente plantearse la siguiente pregunta ¿De qué manera los docentes y estudiantes abordados en esta investigación combinan dichas unidades dispersas para la elaboración de esquemas observacionales relacionados con las prácticas educativas en el marco de la pandemia COVID - 19?

Tal y como se planteó en el marco teórico, para dar respuesta a una pregunta de estas características, cobra relevancia la categoría forma, pues esta hace referencia al resultado de la particular combinación, realizada por el observador, con respecto a las unidades dispersas. En este orden de ideas, los esquemas observacionales se pueden inferir a partir de la distinción de las formas constituidas por los observadores.

En el caso específico de los docentes y estudiantes abordados en este proyecto de investigación, emerge un esquema observacional de carácter relacional que se estructura a partir de las siguientes formas: a) práctica educativa y TIC, b) competencias TIC, c) usabilidad TIC y d) comunicación / interacción / diálogo.

Se afirma que el esquema observacional es de carácter relacional, por cuanto la forma comunicación / interacción /diálogo, funge como enlace entre las formas práctica educativa, competencias TIC y usabilidad TIC, las cuales constituyen un espacio multidimensional de representaciones, significados y sentidos, en la movilidad que ofrece la forma referida, donde la mediatización de las experiencias de aprendizaje, configura una cultura nómada en un entorno de relaciones sociales, (Lévy, 2001). Parece ser que las relaciones que establecen los docentes con las TIC en sus prácticas educativas, cobra relevancia en la medida en que dichas tecnologías permiten relacionarse, interactuar $y$ comunicarse con los otros. (Ferrés, 2014; Lévy, 2004, Orozco, 2004, Prensky, 2011)

En tal sentido, tanto el acceso a los artefactos tecnológicos como la conectividad y la alfabetización digital, se constituyen en el medio que permite la comunicación, la interacción y el diálogo con los demás, sobre todo en un escenario pandémico en el que el contacto físico se ha visto limitado, por lo que las relaciones mediatizadas se han intensificado (Pérez - Tornero, 2020).

El proceso comunicativo se vincula como una acción natural e indispensable en los procesos educativos, con enfoques reticulares para las relaciones e intercambios entre los actores y elementos del sistema comunicacional que rodea el entorno educativo, todo ello debería permitir poner en común los conocimientos, aprendizajes y compartirlo recíprocamente, (Lévy, 2004; Siemens, 2004).

Asimismo, las prácticas educativas se presentan en continua transformación, dados los entornos enriquecidos por tecnologías, información y algoritmos, en los cuales 
se precisa de acciones relevantes en la educación, donde los docentes, centren dichas acciones en el acompañamiento y gestión de los aprendizajes, en la mediación racional y simbólica, y en el pilotaje de los recorridos de aprendizaje; todo esto, desde la interacción y la comunicación, valorando el aprendizaje con otros, (Lévy, 2004).

En particular, las estrategias y metodologías implementadas, en los entornos virtuales, no son distantes de las que se utilizan en los entornos presenciales de formación, dichas estrategias presentan intereses marcados en la dimensión humana para los intercambios entre los participantes de la experiencia educativa, a partir de las interacciones, la comunicación y el diálogo, para así, extender la experiencia $\mathrm{y}$ tejer relaciones permanentes entre los participantes del entorno de formación, todos ellos dados en el vínculo social, para la descentralización de la memoria común en el medio algorítmico. (Gros, 2004; Lévy, 2019; Siemens, 2004)

De esta manera, los docentes y estudiantes, valoran significativamente las interacciones, la comunicación y la relación con los otros, en las prácticas educativas para el desarrollo de habilidades útiles para el aprendizaje y el ecosistema mediático, independientemente de la modalidad para la cual se articulen. Dado que el énfasis en el diseño de experiencias basadas en la interacción social y la participación, favorecen ese elemento movilizador de la práctica educativa, (Gros, 2015).

Lo cual permite inferir que el diseño del aprendizaje se traduce en una metodología móvil para la telepresencia o para la formación presencial, promoviendo el uso apropiado de recursos y tecnologías, la potenciación de interacciones con el propósito de tomar decisiones más informadas en el diseño de intervenciones educativas (Conole, 2013), es decir, el docente en la práctica, podría enfocar también sus esfuerzos en el diseño de experiencias de aprendizaje, que permitan en el vínculo social, la generación de interacciones significativas y recíprocas entre los sujetos participantes. (Kuklinski, 2018).

Es así como, se evidencia que la forma comunicación/interacción/ diálogo, son los enlaces que circulan y activan otras estrategias en las prácticas educativas, donde profesores y estudiantes, en el acervo común de datos, se reúnen e interactúan a través de algoritmos, que permiten el intercambio social en la memoria común que se transforma caóticamente, resultados de la interacción y las habilidades del pensamiento, necesarias tanto en contextos virtuales, como presenciales, estableciendo sistemas de comunicación implícitos a través del medio y un gran número de símbolos disponibles. (Lévy, 2015; Cobo, 2019)

Pareciera una perogrullada, pero el hallazgo más grande fue la emergencia del ser humano, como el centro de la preocupación en las prácticas educativas con telepresencia en época del COVID 19, más allá de los contenidos y la malla curricular, la motivación era el encuentro con el Otro. 


\section{Referencia bibliográfica}

Akhyar, M. (2020). Enhancing Higher-Order Thinking Skills in Vocational Education through Scaffolding Problem Based Learning. Open Engineering, 10(1), 612-619.

Bedoya, O, (2019). Metodología relacional en investigación cualitativa: más allá del análisis, Universidad Tecnológica de Pereira.

Castells, M (2009) Comunicación y Poder. Alianza Editorial. Madrid.

Castells, M. (2014). El impacto de internet en la sociedad: una perspectiva global.C@mbio,19, 127-149.

CEPAL, N. (2020). La educación en tiempos de la pandemia de COVID-19.

Cobo Romaní, J.C. (2009) El concepto de tecnologías de la información. Benchmarking sobre las definiciones de las TIC en la sociedad del conocimiento. Zer Vol 14, Num 27, (295-318)

Cobo, Cristóbal (2019): Acepto las Condiciones: Usos y abusos de las tecnologías digitales, Fundación Santillana, Madrid.

Conole, G. (2013). Las pedagogías de los entornos personales de aprendizaje. En L. Castañeda y J. Adell (Eds.), Entornos Personales de Aprendizaje: Claves para el ecosistema educativo en red ( $\mathrm{pp}$. 185-188). Alcoy: Marfil
Cruz Pérez, Miguel Alejandro, \& Pozo Vinueza., Mónica Alexandra. (2020). Contenido científico en la formación investigativa a través de las TIC en estudiantes universitarios. E-Ciencias de la Información, 10(1), 136-158.

Csikszentmihalyi, M. (1998). Creatividad: el fluir y la psicología del descubrimiento y la invención (pp. 41-71). Barcelona: Paidós.

Ferrés, J. (2014). Las pantallas y el cerebro emocional. Editorial Gedisa.

Gros, B. (2015). La caída de los muros del conocimiento en la sociedad digital y las pedagogías emergentes.

Hodges, Charles; Moore, Stephanie: Lockee, Barb; Trust, Torrey; Bond, Aaron. The Difference Between Emergency Remote Teaching and Online Learning. Educause. March 27, 2020 .

Lévy, P. (2004). Inteligencia colectiva. Por una antropología del ciberespacio. Organización Panamericana de la Salud. Qualitative Research in Education, 4 (3), 323.

Levy, P. (2015). Collective intelligence for educators.

Levy, P. (2015). The Emergence of Reflexive Collective Intelligence, [en línea] https://pierrelevyblog. com/2015/04/14/the-emergenceof-reflexive-collective-intelligencel 
Liesa-Orús, M., Latorre-Cosculluela, C., Vázquez-Toledo, S., \& Sierra-Sánchez, V. (2020). The technological challenge facing higher education professors: Perceptions of ICT tools for developing 21 st century skills. Sustainability, 12(13), 5339.

Luhmann, N. (1995). Social systems. Stanford University Press.

Luhmann, N. (1998). Observations on modernity. Stanford University Press.

Ministerio de Educación de Colombia (2013), "Competencias TIC para el desarrollo profesional del docente", [en línea] https:// www.mineducacion.gov.co/1759/ articles-339097 archivo pdf competencias tic.pdf

Ministerio de Educación de Colombia (2020), "Ministerio de Educación Nacional e Instituciones de Educación Superior fortalecen actividades académicas asistidas con herramientas TIC a través del plan padrino, una oportunidad para el crecimiento del sector" [en línea] https://www.mineducacion. gov.co/portal/salaprensa/ Noticias/396744:Ministerio$\mathrm{de}-\mathrm{Educ}$ acion-Nacionale - In s t i t u c i o n e s-d e EducacionSuperior-fortalecenactividades-academicas-asistidascon-herramientas-TIC-a-travesdel-plan-padrinouna-oportunidadpara-el-cre [fecha de consulta: 12 de junio de 2020].
Orozco, G. (2004). De la enseñanza al aprendizaje: desordenamientos educomunicativos en los tiempos, escenarios y procesos de conocimiento. Revista Nómadas, (21), 120-127 https://bit. ly/2FHyFl4

Oseda Gago, Dulio, Mendivel Geronimo, Ruth Katherine, \& Angoma Astucuri, Miriam. (2020). Estrategias didácticas para el desarrollo de competencias y pensamiento complejo en estudiantes universitarios. Sophia, Colección de Filosofia de la Educación, (29), 235-259. https://dx.doi.org/10.17163/soph. $\underline{\mathrm{n} 29.2020 .08}$

Pardo Kuklinski, Hugo; Cobo, Cristóbal (2020). Expandir la universidad más allá de la enseñanza remota de emergencia Ideas hacia un modelo híbrido post-pandemia. Outliers School. Barcelona.

Rieble-Aubourg, S. y A. Viteri (2020), "COVID-19: ¿Estamos preparados para el aprendizaje en línea?", Nota CIMA, $\mathrm{N}^{\circ}$ 20, Washington, D.C., Banco Interamericano de Desarrollo (BID) [en línea] https:// publications.iadb.org/publications/ spanish/document/Nota-CIMA-20-COV

Presnky, M. (2011). Enseñar a nativos digitales. Ediciones S.M.

Pérez, Tornero, J. (2020). La gran mediatización I. El Tsunami que expropia nuestras vidas. Del confinamiento digital, a la sociedad de la distancia. UOCpress. 
Siemens, G. (2004). Elearnspace. Connectivism: A learning theory for the digital age. Elearnspace. org.

Suárez, C., (2012). De la escuela-lugar a la escuela-nodo. Blog sobre Educación y Virtualidad. http:// educacion-virtualidad.blogspot. com/2012/03/de-la-escuela-lugarla-escuela-nodo.html

Tornero, J. M. P. (2020). La gran mediatización I: el tsunami que expropia nuestras vidas: del confinamiento social a la sociedad a distancia. Fundació per la Universitat Oberta de Catalunya.

UNESCO (Organización de las Naciones Unidas para la Educación, la Ciencia y la Cultura) (2019), Marco de competencias de los docentes en materia de TIC, París [en línea] https://unesdoc. unesco. org/ark:/48223/pf0000371024.

Zabala Vidiella, A. (1995). La práctica educativa. Como Enseñar. Editorial Graó España. 